TRANSACTIONS OF THE

AMERICAN MATHEMATICAL SOCIETY

Volume 361, Number 12, December 2009, Pages 6501-6519

S 0002-9947(09)04682-0

Article electronically published on July 24, 2009

\title{
CALABI-YAU OBJECTS IN TRIANGULATED CATEGORIES
}

\author{
CLAUDE CIBILS AND PU ZHANG
}

\begin{abstract}
We introduce the Calabi-Yau (CY) objects in a Hom-finite KrullSchmidt triangulated $k$-category, and notice that the structure of the minimal, consequently all the CY objects, can be described. The relation between indecomposable CY objects and Auslander-Reiten triangles is provided. Finally we classify all the CY modules of self-injective Nakayama algebras, determining in this way the self-injective Nakayama algebras admitting indecomposable CY modules.
\end{abstract}

\section{INTRODUCTION}

Calabi-Yau (CY) categories have been introduced by Kontsevich $\mathrm{Ko}$. They provide a new insight and a wide framework for topics as in mathematical physics ([Co]), non-commutative geometry ([B], Gin1, Gin2]), and representation theory of Artin algebras ( $\mathrm{BS}, \overline{\mathrm{ES}}, \mathrm{IR}, \overline{\mathrm{Ke}}, \mathrm{KR} 1, \mathrm{KR} 2)$.

Triangulated categories with Serre dualities ([BK], $[\mathrm{RV}]$ ) and CY categories have important global naturality. On the other hand, even in non-CY categories, inspired by $[\mathrm{Ko}$, one can introduce $\mathrm{CY}$ objects. It turns out that they arise naturally in non-CY categories and enjoy "local naturality" and interesting properties (Proposition 4.4, Theorems 3.2, 4.2, 5.5 and 6.1).

The first aim of this paper is to study the properties of such objects in a Homfinite Krull-Schmidt triangulated $k$-category with Serre functor $F$. We give the relation between indecomposable $\mathrm{CY}$ objects and the Auslander-Reiten triangles $(\S 3)$, and describe all the $d$-th CY objects via the minimal ones, which are exactly the direct sum of all the objects in finite $\langle[-d] \circ F\rangle$-orbits of $\operatorname{Ind}(\mathcal{A})(\S 4)$. We classify all the $d$-th CY modules of self-injective Nakayama algebras for any integer $d(\S 5)$. Finally, we determine all the self-injective Nakayama algebras which admit indecomposable CY modules. In particular, this recovers the algebras whose stable categories are Calabi-Yau ( $(6)$, included in the work of Bialkowski and Skowroński BS. Note that the CY modules are invariant under stable equivalences between self-injective algebras, with very few exceptions (Proposition 3.1). Consequently our results on self-injective Nakayama algebras extend to the one on the wreathlike algebras [GR], which contains the Brauer tree algebras [J].

Received by the editors November 16, 2007.

2000 Mathematics Subject Classification. Primary 18E30; Secondary 16G20, 16G70, 16E30, $18 \mathrm{G} 20$.

Key words and phrases. Serre functor, Calabi-Yau object, Auslander-Reiten triangle, stable module category, self-injective Nakayama algebra.

The second author was supported by the Chinese Natural Science Foundation for Distinguished Young Scholars (Grant No. 10725104) and the CNRS of France.

$\mathrm{Pu}$ Zhang is the corresponding author for this paper.

(C)2009 American Mathematical Society Reverts to public domain 28 years from publication 
This also raises an immediate question. Let $\mathcal{A}$ be a Hom-finite Krull-Schmidt triangulated $k$-category with a Serre functor. If all objects are $d$-th CY with the same $d$, whether $\mathcal{A}$ is a Calabi-Yau category?

\section{BACKGROUNDS AND PRELIMINARIES}

2.1. Let $k$ be a field and $\mathcal{A}$ a Hom-finite $k$-category. Recall from Bondal and Kapranov [BK] that a $k$-linear functor $F: \mathcal{A} \rightarrow \mathcal{A}$ is a right Serre functor if there exist $k$-isomorphisms

$$
\eta_{A, B}: \operatorname{Hom}_{\mathcal{A}}(A, B) \longrightarrow D \operatorname{Hom}_{\mathcal{A}}(B, F A), \quad \forall A, B \in \mathcal{A},
$$

which are natural both in $A$ and $B$, where $D=\operatorname{Hom}_{k}(-, k)$. Such an $F$ is unique up to a natural isomorphism, and fully faithful; if it is an equivalence, then a quasiinverse $F^{-1}$ is a left Serre functor; in this case we call $F$ a Serre functor. Note that $\mathcal{A}$ has a Serre functor if and only if it has both a right and a left Serre functor. See Reiten and Van den Bergh [RV].

For triangulated categories we refer to $[\mathrm{Har},[\mathrm{V}]$, and $[\mathrm{N}]$. Let $\mathcal{A}$ be a Hom-finite triangulated $k$-category. Following Happel Hap1, an Auslander-Reiten triangle $X \stackrel{f}{\longrightarrow} Y \stackrel{g}{\longrightarrow} Z \stackrel{h}{\longrightarrow} X[1]$ of $\mathcal{A}$ is a distinguished triangle satisfying:

(AR1) $X$ and $Z$ are indecomposable.

(AR2) $h \neq 0$.

(AR3) If $t: Z^{\prime} \longrightarrow Z$ is not a retraction, then there exists $t^{\prime}: Z^{\prime} \longrightarrow Y$ such that $t=g t^{\prime}$.

Note that (AR3) is equivalent to

(AR4) If $Z^{\prime}$ is indecomposable and $t: Z^{\prime} \longrightarrow Z$ is a non-isomorphism, then $h t=0$.

Under (AR1) and (AR2), (AR3) is equivalent to

$\left(\mathrm{AR} 3^{\prime}\right)$ If $s: X \longrightarrow X^{\prime}$ is not a section, then there exists $s^{\prime}: Y \longrightarrow X^{\prime}$ such that $s=s^{\prime} f$.

Also, $\left(\mathrm{AR} 3^{\prime}\right)$ is equivalent to

$\left(\mathrm{AR} 4^{\prime}\right)$ If $X^{\prime}$ is indecomposable and $s: X \longrightarrow X^{\prime}$ is a non-isomorphism, then $s \circ h[-1]=0$.

In an Auslander-Reiten triangle $X \longrightarrow Y \longrightarrow Z \longrightarrow X$ [1], the object $X$ is uniquely determined by $Z$. Write $X=\tau_{\mathcal{A}} Z$. In general $\tau_{\mathcal{A}}$ is not a functor. By definition $\mathcal{A}$ has right Auslander-Reiten triangles if there exists an Auslander-Reiten triangle $X \longrightarrow Y \longrightarrow Z \longrightarrow X[1]$ for any indecomposable $Z$, and $\mathcal{A}$ has Auslander-Reiten triangles if $\mathcal{A}$ has right and left Auslander-Reiten triangles. We refer to Hap1, XZ] and $\mathrm{A}$ for the Auslander-Reiten quiver of a triangulated category.

A Hom-finite $k$-category is Krull-Schmidt if the endomorphism algebra of any indecomposable is local. In this case any object is uniquely decomposed into a direct sum of indecomposables, up to isomorphisms and up to the order of indecomposable direct summands (Ringel $[\mathrm{R}$, p. 52).

Let $\mathcal{A}$ be a Hom-finite Krull-Schmidt triangulated $k$-category. Theorem I.2.4 in [RV] says that $\mathcal{A}$ has a right Serre functor $F$ if and only if $\mathcal{A}$ has right AuslanderReiten triangles. In this case, $F$ coincides with [1] $\circ \tau_{\mathcal{A}}$ on objects, up to isomorphisms.

2.2. Let $\mathcal{A}$ be a Hom-finite triangulated $k$-category with Serre functor $F$. Denote by [1] the shift functor of $\mathcal{A}$. Following Kontsevich [Ko, $\mathcal{A}$ is a Calabi-Yau category if there is a natural isomorphism $F \cong[d]$ of functors for some $d \in \mathbb{Z}$. 
Denote by $o([1])$ the order of [1]. If $o([1])=\infty$, then the integer $d$ above is unique and is called the $C Y$ dimension of $\mathcal{A}$. If $o([1])$ is finite, then we call the minimal non-negative integer $d$ such that $F \cong[d]$ the $C Y$ dimension of $\mathcal{A}$. Denote by $\mathrm{CY} \operatorname{dim}(\mathcal{A})$ the $\mathrm{CY}$ dimension of $\mathcal{A}$.

For example, if $A$ is a symmetric algebra and $\mathcal{P}$ is the category of projective modules, then the homotopy category $K^{b}(\mathcal{P})$ is of $\mathrm{CY}$ dimension 0 . Moreover, if $\mathcal{A}$ is of CY dimension $d$, then $\operatorname{Ext}_{\mathcal{A}}^{i}(X, Y) \cong D \circ \operatorname{Ext}_{\mathcal{A}}^{d-i}(Y, X), X, Y \in \mathcal{A}, i \in \mathbb{Z}$, where $\operatorname{Ext}_{\mathcal{A}}^{i}(X, Y):=\operatorname{Hom}_{\mathcal{A}}(X, Y[i])$. Thus, if $A$ is a CY algebra (B], Gin2]), i.e. the bounded derived category $D^{b}(A$-mod) is Calabi-Yau of CY dimension $d$, then gl. $\operatorname{dim}(A$-mod $)=d($ see $[\mathbf{B}])$.

2.3. Let $\mathcal{A}$ and $\mathcal{B}$ be triangulated categories. $A$ triangle functor from $\mathcal{A}$ to $\mathcal{B}$ is a pair $\left(F, \eta^{F}\right)$, where $F: \mathcal{A} \rightarrow \mathcal{B}$ is an additive functor and $\eta^{F}: F \circ[1] \longrightarrow[1] \circ F$ is a natural isomorphism, such that if $X \stackrel{f}{\longrightarrow} Y \stackrel{g}{\longrightarrow} Z \stackrel{h}{\longrightarrow} X[1]$ is a distinguished triangle of $\mathcal{A}$, then $F X \stackrel{F f}{\longrightarrow} F Y \stackrel{F g}{\longrightarrow} F Z \stackrel{\eta_{X}^{F} \circ F h}{\longrightarrow}(F X)[1]$ is a distinguished triangle of $\mathcal{B}$. Triangle functors $\left(F, \eta^{F}\right)$ and $\left(G, \eta^{G}\right)$ are natural isomorphic if there is a natural isomorphism $\xi: F \longrightarrow G$ such that the following diagram commutes for any $A \in \mathcal{A}$ :

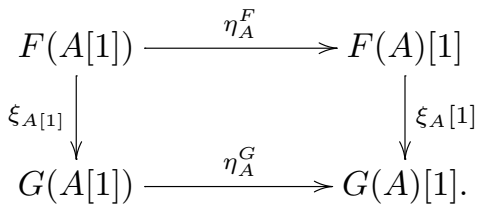

As Keller pointed out, the pair $\left([n],(-1)^{n} \operatorname{Id}_{[n+1]}\right): \mathcal{A} \longrightarrow \mathcal{A}$ is a triangle functor for $n \in \mathbb{Z}$; however, $\left([n], \operatorname{Id}_{[n+1]}\right)$ may not.

We need the following important result. A nice proof given by Van den Bergh is in the Appendix of $[\mathrm{B}$.

Lemma 2.1 (Bondal-Kapranov [BK]; Van den Bergh [B]). Let $F$ be a Serre functor of a Hom-finite triangulated $k$-category $\mathcal{A}$. Then there exists a natural isomorphism $\eta^{F}: F \circ[1] \longrightarrow[1] \circ F$ such that $\left(F, \eta^{F}\right): \mathcal{A} \longrightarrow \mathcal{A}$ is a triangle functor.

From [ $\mathrm{Ke}, 8.1]$ and $[\mathrm{B}, \mathrm{A} .5 .1]$ one has the following

Proposition 2.2 (Keller; Van den Bergh). Let $\mathcal{A}$ be a Hom-finite triangulated $k$-category with Serre functor $F$. Then $\mathcal{A}$ is a Calabi-Yau category if and only if there exists a natural isomorphism $\eta^{F}: F \circ[1] \longrightarrow[1] \circ F$, such that $\left(F, \eta^{F}\right)$ is a triangle functor and $\left(F, \eta^{F}\right) \longrightarrow\left([d],(-1)^{d} \operatorname{Id}_{[d+1]}\right)$ is a natural isomorphism of triangle functors, for some integer $d$.

Proof. For convenience we justify the "only if" part. By assumption we have a natural isomorphism $\xi: F \cong[d]$. Define $\eta_{A}^{F}: F(A[1]) \longrightarrow(F A)[1]$ for $A \in \mathcal{A}$ by $\eta_{A}^{F}:=(-1)^{d}\left(\xi_{A}\right)^{-1}[1] \circ \xi_{A[1]}$. Then $\xi_{A}[1] \circ \eta_{A}^{F}=(-1)^{d} \xi_{A[1]}$. The naturality of $\eta^{F}$ : $F \circ[1] \longrightarrow[1] \circ F$ follows from the one of $\xi$. It remains to show that $\left(F, \eta^{F}\right): \mathcal{A} \rightarrow \mathcal{A}$ is a triangle functor. Let $X \stackrel{f}{\longrightarrow} Y \stackrel{g}{\longrightarrow} Z \stackrel{h}{\longrightarrow} X[1]$ be a distinguished triangle. Since $X[d] \stackrel{f[d]}{\longrightarrow} Y[d] \stackrel{g[d]}{\longrightarrow} Z[d] \stackrel{(-1)^{d} h[d]}{\longrightarrow} X[d+1]$ is a distinguished triangle, it 
suffices to prove that the following diagram is commutative:

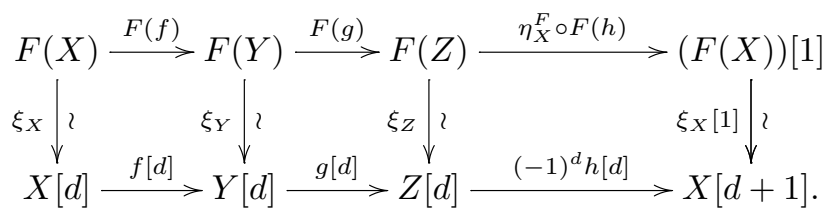

By the naturality of $\xi$ the first and the second square are commutative. We also have

$$
\xi_{X}[1] \circ \eta_{X}^{F} \circ F(h)=(-1)^{d} \xi_{X[1]} \circ F(h)=(-1)^{d} h[d] \circ \xi_{Z} .
$$

2.4. Let $A$ be a self-injective $k$-algebra, $A$-mod the category of finite-dimensional left $A$-modules, and $A$-mod the stable category of $A$-mod modulo projective modules. Then the Nakayama functor $\mathcal{N}:=D(A) \otimes_{A}-$, Heller's syzygy functor $\Omega$, and the Auslander-Reiten translate $\tau \cong \Omega^{2} \circ \mathcal{N} \cong \mathcal{N} \circ \Omega^{2}$ (ARS, p. 126) are endo-equivalences of $A$-mod [ARS, Chap. IV]. Note that $A$-mod is a Hom-finite Krull-Schmidt triangulated $k$-category with [1] $=\Omega^{-1}$ Hap1, p. 16]. By the binaturality of the Auslander-Reiten isomorphisms $\mathrm{AR}$.

$$
\underline{\operatorname{Hom}}(X, Y) \cong D \circ \operatorname{Ext}_{A}^{1}(Y, \tau X) \cong D \circ \underline{\operatorname{Hom}}(Y,[1] \circ \tau X),
$$

where $\underline{\operatorname{Hom}}(X, Y):=\operatorname{Hom}_{A-\bmod }(X, Y)$, one gets the Serre functor $F:=[1] \circ \tau \cong$ $\Omega \circ \mathcal{N}$ of $A$-mod. It follows that $A$-mod is Calabi-Yau if and only if $\mathcal{N} \cong \Omega^{-(d+1)}$ for some $d[\mathrm{Ke}, 8.3]$. In this case denote by $\mathrm{CY} \operatorname{dim}(A)$ the CY dimension of $A$-mod. Note that $\Omega, F, \mathcal{N}, \tau$ are pairwise commutative as functors of $A$-mod. This follows from Lemma 2.1 .

2.5. Let $A$ be a finite-dimensional $k$-algebra. Recall that $A$ is a Nakayama algebra if any indecomposable is uniserial, i.e. it has a unique composition series ARS, p. 197]. In this case $A$ is representation-finite. If $k$ is algebraically closed, then any connected self-injective Nakayama algebra is Morita equivalent to $\Lambda(n, t), n \geq$ $1, t \geq 2$ [GR, p. 243], which is defined below.

Let $\mathbb{Z}_{n}$ be the cyclic quiver with vertices indexed by the cyclic group $\mathbb{Z} / n \mathbb{Z}$ of order $n$, and with arrows $a_{i}: i \longrightarrow i+1, \forall i \in \mathbb{Z} / n \mathbb{Z}$. Let $k \mathbb{Z}_{n}$ be the path algebra of the quiver $\mathbb{Z}_{n}, J$ the ideal generated by all arrows, and $\Lambda=\Lambda(n, t):=k \mathbb{Z}_{n} / J^{t}$ with $t \geq 2$. Denote by $\gamma_{i}^{l}$ the path starting at vertex $i$ and of length $l$, and $e_{i}:=\gamma_{i}^{0}$. We write the conjunction of paths from right to left. Then $\left\{\gamma_{i}^{l} \mid 0 \leq i \leq\right.$ $n-1,0 \leq l \leq t-1\}$ is a basis of $\Lambda$, while $\left\{P(i):=\Lambda e_{i} \mid 0 \leq i \leq n-1\right\}$ is the set of pairwise non-isomorphic indecomposable projective modules and $\{I(i):=$ $\left.D\left(e_{i} \Lambda\right) \mid 0 \leq i \leq n-1\right\}$ is the set of pairwise non-isomorphic indecomposable injective modules, with $P(i) \cong I(i+t-1)$. Note that $\Lambda$ is a Frobenius algebra; and that $\Lambda$ is symmetric if and only if $n \mid(t-1)$. Write $S(i):=P(i) / \operatorname{rad} P(i)$ and $S_{i}^{l}:=\Lambda \gamma_{i+l-t}^{t-l}$. Then $S_{i}^{l}$ is the indecomposable with top $S(i)$ and the Loewy length $l$, and $\left\{S_{i}^{l} \mid 0 \leq i \leq n-1,1 \leq l \leq t\right\}$ is the set of pairwise non-isomorphic indecomposable modules with $S_{i}^{t}=P(i)$ and $\operatorname{soc}\left(S_{i}^{l}\right)=S(i+l-1)$. For the Auslander-Reiten quiver of $\Lambda$ see [GR, Section 2, and [ARS, p. 197. In particular, the stable Auslander-Reiten quiver of $\Lambda$ is $\mathbb{Z} A_{t-1} /\left\langle\tau^{n}\right\rangle$. 


\section{Indecomposable CAlabi-Yau objects}

The purpose of this section is to introduce the Calabi-Yau objects and to give the relation between indecomposable Calabi-Yau objects and Auslander-Reiten triangles.

3.1. Let $\mathcal{A}$ be a Hom-finite triangulated $k$-category. A non-zero object $X$ is called a Calabi-Yau object if there exists a natural isomorphism

$$
\operatorname{Hom}_{\mathcal{A}}(X,-) \cong D \circ \operatorname{Hom}_{\mathcal{A}}(-, X[d])
$$

for some integer $d$.

By the Yoneda Lemma, such a $d$ is unique up to a multiple of the relative order $o\left([1]_{X}\right)$ of $[1]$ with respect to $X$. Recall that $o\left([1]_{X}\right)$ is the minimal positive integer such that $X\left[o\left([1]_{X}\right)\right] \cong X$; otherwise $o\left([1]_{X}\right)=\infty$. If $o\left([1]_{X}\right)=\infty$, then $d$ in (3.1) is unique and is called the $C Y$ dimension of $X$. If $o\left([1]_{X}\right)$ is finite, then the minimal non-negative integer $d$ in (3.1) is called the $C Y$ dimension of $X$. We denote $\mathrm{CY} \operatorname{dim}(X)$ as the CY dimension. Thus, if $o([1])<\infty$, then $o\left([1]_{X}\right) \mid o([1])$ and $0 \leq \mathrm{CY} \operatorname{dim}(X)<o\left([1]_{X}\right)$

Let $A$ be a finite-dimensional self-injective algebra. An $A$-module $M$ without projective direct summands is called a Calabi-Yau module of CY dimension $d$ if it is a Calabi-Yau object of $A$-mod with $\mathrm{CY} \operatorname{dim}(M)=d$.

Note that $\mathrm{CY} \operatorname{dim}(X)$ is usually not easy to determine. In case (3.1) holds for some $d$, we say that $X$ is a $d$-th $C Y$ object. Of course, if $o\left([1]_{X}\right)<\infty$, then $o\left([1]_{X}\right) \mid(d-\mathrm{CY} \operatorname{dim}(X)) \geq 0$.

If $\mathcal{A}$ has a right Serre functor $F$, then by the Yoneda Lemma a non-zero object $X$ is a $d$-th $\mathrm{CY}$ object if and only if $F(X) \cong X[d]$, or equivalently, $F(X)[-d] \cong X$. Thus, a non-zero $A$-module $M$ without projective direct summands is a $d$-th CY module if and only if $\mathcal{N}(M) \cong \Omega^{-(d+1)}(M)$ in $A$-mod (in fact, this isomorphism can be taken in $A$-mod).

3.2. We have the following basic property.

Proposition 3.1. (i) The Calabi-Yau property for a category or an object is invariant under triangle-equivalences.

(ii) The Calabi-Yau property for a module is "usually" invariant under stable equivalences between self-injective algebras. Precisely, let $A$ and $B$ be self-injective algebras, $G: A \underline{\text { mod }} \longrightarrow$ B-mod a stable equivalence, and $X$ a $C Y$ A-module of dimension d. If $\bar{A} \Lambda(n, 2)$, or if $A$ and $B$ are symmetric algebras, then $G(X)$ is a CY B-module of dimension $d$.

Proof. (i) Let $G: \mathcal{A} \longrightarrow \mathcal{B}$ be a triangle-equivalence, and $\mathcal{A}$ be a Calabi-Yau category with $F_{\mathcal{A}} \cong[d]$, where $F_{\mathcal{A}}$ is the Serre functor. Clearly $F_{\mathcal{B}}:=G \circ F_{\mathcal{A}} \circ G^{-1}$ is a Serre functor of $\mathcal{B}$ (if $\mathcal{B}$ already has one, then it is naturally isomorphic to $F_{\mathcal{B}}$ ). By the natural isomorphism $\left(\xi^{G}\right)^{d}: G \circ[d] \longrightarrow[d] \circ G$, which is the composition $G \circ[d] \longrightarrow[1] \circ G \circ[d-1] \longrightarrow \cdots \longrightarrow[d] \circ G$, we see that $\mathcal{B}$ is a Calabi-Yau category with $F_{\mathcal{B}} \cong[d]$. If $X$ is a Calabi-Yau object with a natural isomorphism $\eta$ as in (3.1), then we have the natural isomorphism $\operatorname{Hom}_{\mathcal{B}}\left(-,\left(\xi^{G}\right)_{X}^{d}\right) \circ G \circ \eta \circ G^{-1}$ : $\operatorname{Hom}_{\mathcal{B}}(G(X),-) \cong D \circ \operatorname{Hom}_{\mathcal{B}}(-,(G X)[d])$, which implies that $G(X)$ is a CalabiYau object of $\mathcal{B}$.

(ii) Recall that an equivalence $G: A \underline{-\bmod } \longrightarrow B$-mod of categories is called a stable equivalence. Note that in general $G$ is not induced by an exact functor 
(cf. ARS, p. 339), hence $G$ may not be a triangle-equivalence (cf. Hap1, Lemma 2.7 , p. 22 ; note that the converse of Lemma 2.7 is also true). One may assume that $A$ is connected. If $A \nsubseteq \Lambda(n, 2)$, or if $A$ and $B$ are symmetric algebras, then by Corollary 1.7 and Proposition 1.12 in [ARS], p. 344, we know that $G$ commutes with $\tau$ and $\Omega$ on modules. Hence we have isomorphism

$$
\Omega_{B}^{-1} \circ \tau_{B}(G(X)) \cong G\left(\Omega_{A}^{-1} \circ \tau_{A}\right)(X) \cong G\left(\Omega_{A}^{-d}(X)\right) \cong \Omega_{B}^{-d}(G(X)),
$$

which implies that $G(X)$ is a Calabi-Yau $B$-module of CY dimension $d$.

It seems that the Calabi-Yau property for the stable category is also invariant under stable equivalence $G$ between self-injective algebras. However, this needs natural isomorphisms between $G$ and $\tau$, and $G$ and $\Omega$, which are not clear to us.

3.3. The main result of this section is as follows.

Theorem 3.2. Let $\mathcal{A}$ be a Hom-finite Krull-Schmidt triangulated $k$-category, and $X$ an indecomposable object of $\mathcal{A}$. Then $X$ is a d-th $C Y$ object if and only if there exists an Auslander-Reiten triangle of the form

$$
X[d-1] \stackrel{f}{\longrightarrow} Y \stackrel{g}{\longrightarrow} X \stackrel{h}{\longrightarrow} X[d] .
$$

Moreover, $Y$ is also a d-th $C Y$ object.

3.4. The proof of the first part of Theorem 3.2 follows an argument of Reiten and Van den Bergh in [RV]. For convenience we include a complete proof.

Lemma 3.3. Let $\mathcal{A}$ be a Hom-finite Krull-Schmidt triangulated $k$-category, and $X$ a non-zero object of $\mathcal{A}$. Then $X$ is a $d$-th $C Y$ object if and only if for any indecomposable $Z$ there exists a non-degenerate bilinear form

$$
(-,-)_{Z}: \operatorname{Hom}_{\mathcal{A}}(X, Z) \times \operatorname{Hom}_{\mathcal{A}}(Z, X[d]) \longrightarrow k
$$

such that for any $u \in \operatorname{Hom}_{\mathcal{A}}(X, Z), v \in \operatorname{Hom}_{\mathcal{A}}(Z, W)$, and $w \in \operatorname{Hom}_{\mathcal{A}}(W, X[d])$, there holds

$$
(u, w v)_{Z}=(v u, w)_{W}
$$

Proof. If $X$ is a $d$-th CY object, then we have

$$
\eta_{Z}: \operatorname{Hom}_{\mathcal{A}}(X, Z) \cong D \circ \operatorname{Hom}_{\mathcal{A}}(Z, X[d]), \forall Z \in \mathcal{A},
$$

which are natural in $Z$. Each isomorphism $\eta_{Z}$ induces a non-degenerate bilinear form $(-,-)_{Z}$ in $(3.3)$ by $(u, z)_{Z}:=\eta_{Z}(u)(z)$, and (3.4) follows from the naturality of $\eta_{Z}$ in $Z$. Conversely, if we have (3.3) and (3.4) for any indecomposable $Z$, then we have isomorphism $\eta_{Z}: \operatorname{Hom}_{\mathcal{A}}(X, Z) \cong D \circ \operatorname{Hom}_{\mathcal{A}}(Z, X[d])$ given by $\eta_{Z}(u)(z):=(u, z)_{Z}, \forall z \in \operatorname{Hom}_{\mathcal{A}}(Z, X[d])$. By (3.4) $\eta_{Z}$ are natural in $Z$. Since $\mathcal{A}$ is Krull-Schmidt, it follows that we have isomorphisms $\eta_{Z}$ for any $Z$ which are natural in $Z$. This means that $X$ is a $d$-th CY object.

The following Lemma in $[\mathrm{RV}]$ will be used.

Lemma 3.4 ([RV], Sublemma I.2.3). Let $\mathcal{A}$ be a Hom-finite Krull-Schmidt triangulated $k$-category, $\tau_{\mathcal{A}}(X) \longrightarrow Y \longrightarrow X \stackrel{h}{\longrightarrow} \tau_{\mathcal{A}}(X)[1]$ an Auslander-Reiten triangle of $\mathcal{A}$, and $Z$ an indecomposable in $\mathcal{A}$. Then

(i) For any non-zero $z \in \operatorname{Hom}_{\mathcal{A}}\left(Z, \tau_{\mathcal{A}}(X)[1]\right)$ there exists $u \in \operatorname{Hom}_{\mathcal{A}}(X, Z)$ such that $z u=h$. 
(ii) For any non-zero $u \in \operatorname{Hom}_{\mathcal{A}}(X, Z)$ there exists $z \in \operatorname{Hom}_{\mathcal{A}}\left(Z, \tau_{\mathcal{A}}(X)[1]\right)$ such that $z u=h$.

In a Hom-finite Krull-Schmidt triangulated $k$-category without Serre functor (e.g., by Hap2 and [RV], if gl. $\operatorname{dim}(A)=\infty$, then $D^{b}$ (A-mod) has no Serre functor), one may use the generalized Serre functor introduced by Chen [Ch.

Lemma 3.5 (Chen $\mathrm{Ch}$ ). Let $\mathcal{A}$ be a Hom-finite Krull-Schmidt triangulated $k$ category. Consider the full subcategories of $\mathcal{A}$ given by

$$
\mathcal{A}_{r}:=\left\{X \in \mathcal{A} \mid D \circ \operatorname{Hom}_{\mathcal{A}}(X,-) \text { is representable }\right\}
$$

and

$$
\mathcal{A}_{l}:=\left\{X \in \mathcal{A} \mid D \circ \operatorname{Hom}_{\mathcal{A}}(-, X) \text { is representable }\right\} .
$$

Then both $\mathcal{A}_{r}$ and $\mathcal{A}_{l}$ are thick triangulated subcategories of $\mathcal{A}$. Moreover, one has

(i) There is a unique $k$-functor $S: \mathcal{A}_{r} \longrightarrow \mathcal{A}_{l}$ which is an equivalence, such that there are natural isomorphisms

$$
\operatorname{Hom}_{\mathcal{A}}(X,-) \simeq D \circ \operatorname{Hom}_{\mathcal{A}}(-, S(X)), \quad \forall X \in \mathcal{A}_{r},
$$

which are natural in $X . S$ is called the generalized Serre functor, with range $\mathcal{A}_{r}$ and domain $\mathcal{A}_{l}$.

(ii) There exists a natural isomorphism $\eta^{S}: S \circ[1] \longrightarrow[1] \circ S$ such that the pair $\left(S, \eta^{S}\right): \mathcal{A}_{r} \longrightarrow \mathcal{A}_{l}$ is a triangle-equivalence.

In this terminology, a non-zero object $X$ is a $d$-th CY object if and only if $X \in \mathcal{A}_{r}$ and $S(X) \cong X[d]$, by (3.5) and the Yoneda Lemma.

3.5. Proof of Theorem 3.2. Let $X$ be an indecomposable $d$-th CY object. By Lemma 3.3 we have a non-degenerate bilinear from $(-,-)_{X}: \operatorname{Hom}_{\mathcal{A}}(X, X) \times$ $\operatorname{Hom}_{\mathcal{A}}(X, X[d]) \longrightarrow k$. It follows that there exists $0 \neq h \in \operatorname{Hom}_{\mathcal{A}}(X, X[d])$ such that $\left(\operatorname{radHom}_{\mathcal{A}}(X, X), h\right)_{X}=0$. Embed $h$ into a distinguished triangle as in (3.2). We claim that it is an Auslander-Reiten triangle. For this it remains to prove (AR4) in $\S 2.1$. Let $X^{\prime}$ be indecomposable and $t: X^{\prime} \longrightarrow X$ a non-isomorphism. Then by (3.4) for any $u \in \operatorname{Hom}_{\mathcal{A}}\left(X, X^{\prime}\right)$ we have $(u, h t)_{X^{\prime}}=(t u, h)_{X}=0$. Since $(-,-)_{X^{\prime}}$ is non-degenerate, it follows that $h t=0$.

Conversely, let (3.2) be an Auslander-Reiten triangle. In order to prove that $X$ is a $d$-th CY object, by Lemma 3.3 it suffices to prove that for any indecomposable $Z$ there exists a non-degenerate bilinear form $(-,-)_{Z}$ as in (3.3) satisfying (3.4). For this, choose an arbitrary linear function $\operatorname{tr} \in D \circ \operatorname{Hom}_{\mathcal{A}}(X, X[d])$ such that $\operatorname{tr}(h) \neq 0$, and define $(u, z)_{Z}=\operatorname{tr}(z u)$. Then (3.4) is automatically satisfied. It remains to prove that $(-,-)_{Z}$ is non-degenerate. In fact, for any $0 \neq z \in \operatorname{Hom}_{\mathcal{A}}(Z, X[d])$, by Lemma 3.4 there exists $u \in \operatorname{Hom}_{\mathcal{A}}(X, Z)$ such that $z u=h$. So $(u, z)_{Z}=\operatorname{tr}(z u)=$ $\operatorname{tr}(h) \neq 0$. Similarly, for any $0 \neq u \in \operatorname{Hom}_{\mathcal{A}}(X, Z)$ we have $z \in \operatorname{Hom}_{\mathcal{A}}(Z, X[d])$ such that $(u, z)_{Z} \neq 0$. This proves the non-degenerateness of $(-,-)_{Z}$.

Now we prove that $Y$ in (3.2) is also a $d$-th CY object. We make use of the generalized Serre functor in $\mathrm{Ch}$ (For the reader who prefers Serre functor, one can assume the existence, and use Lemma 2.1). Since $X, X[d-1] \in \mathcal{A}_{r}$, it follows from Lemma 3.5 that $Y \in \mathcal{A}_{r}$. Applying the generalized Serre functor $\left(S, \eta^{S}\right)$ to (3.2) we get the distinguished triangle (by Lemma 3.5(ii))

$$
S(X[d-1]) \stackrel{S(f)}{\longrightarrow} S(Y) \stackrel{S(g)}{\longrightarrow} S(X) \stackrel{\eta_{X[d-1]}^{S} S(h)}{\longrightarrow} S(X[d-1])[1] .
$$


Also, we have the Auslander-Reiten triangle

$$
X[2 d-1] \stackrel{f[d]}{\longrightarrow} Y[d] \stackrel{g[d]}{\longrightarrow} X[d] \stackrel{(-1)^{d} h[d]}{\longrightarrow} X[2 d] .
$$

Since $X$ is a $d$-th CY object it follows that we have an isomorphism $w: S(X) \longrightarrow$ $X[d]$. Note that $S(h) \neq 0$ means that $S(g)$ is not a retraction ([Hap1, p. 7). Thus, by (AR3) there exists $v: S(Y) \longrightarrow Y[d]$ such that $w \circ S(g)=g[d] \circ v$. By the definition of a triangulated category we get $u: S(X[d-1]) \longrightarrow X[2 d-1]$ such that the following diagram is commutative:

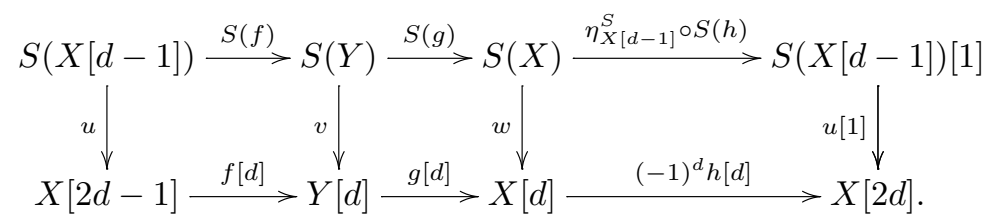

We claim that $u: S(X[d-1]) \longrightarrow X[d]$ is an isomorphism. Hence by the property of a triangulated category we know that $v: S(Y) \longrightarrow Y[d]$ is also an isomorphism, i.e. $Y$ is a $d$-th $\mathrm{CY}$ object.

Otherwise $u: S(X[d-1]) \longrightarrow X[2 d-1]$ is not an isomorphism. Note that $S$ is only defined on $\mathcal{A}_{r}$; it follows that we do not know if $(*)$ is an Auslander-Reiten triangle.

Since $X[d-1]$ is a $d$-th CY object, it follows that we have an isomorphism $\alpha: X[2 d-1] \longrightarrow S(X[d-1])$; hence $\alpha \circ u \in \operatorname{Hom}_{\mathcal{A}}(S(X[d-1]), S(X[d-1]))$. So we have $u^{\prime} \in \operatorname{Hom}_{\mathcal{A}}(X[d-1], X[d-1])$ such that $\alpha \circ u=S\left(u^{\prime}\right)$, and $u^{\prime}$ is also a nonisomorphism. Since $X[d-1] \stackrel{f}{\longrightarrow} Y \stackrel{g}{\longrightarrow} X \stackrel{h}{\longrightarrow} X[d]$ is an Auslander-Reiten triangle it follows from $\left(\mathrm{AR}^{\prime}\right)$ that $u^{\prime} \circ h[-1]=0$, or equivalently, $S\left(u^{\prime}\right) \circ S(h[-1])=0$ (note that $h[-1] \in \mathcal{A}_{r}$ ). Thus we have

$$
u[1] \circ S(h[-1])[1] \circ \eta_{X[-1]}^{S}=0,
$$

where $\eta_{X[-1]}^{S}: S(X) \longrightarrow S(X[-1])[1]$ is an isomorphism. By the naturality of $\eta^{S}$ we have the commutative diagram

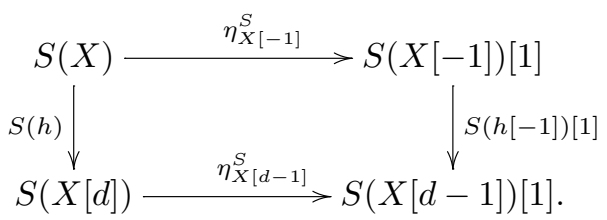

It follows that we have $u[1] \circ \eta_{X[d-1]}^{S} \circ S(h)=0$, and hence by the commutative diagram (**) we get a contradiction: $(-1)^{d} h[d] \circ w=u[1] \circ \eta_{X[d-1]}^{S} \circ S(h)=0$. This completes the proof.

Remark 3.6. Let $\mathcal{A}$ be a Hom-finite Krull-Schmidt triangulated $k$-category. If every indecomposable $X$ in $\mathcal{A}$ is a $d_{X}$-th $\mathrm{CY}$ object, then $\mathcal{A}$ has a Serre functor $F$ with $F(X) \cong X\left[d_{X}\right]$.

In fact, by Theorem $3.2 \mathcal{A}$ has right and left Auslander-Reiten triangles, and then by Theorem I.2.4 in $\mathrm{RV} \mathcal{A}$ has the Serre functor $F$. By Proposition I.2.3 in [RV] and (3.2) we have $F(X) \cong \tau_{\mathcal{A}}(X)[1]=X\left[d_{X}-1\right][1]=X\left[d_{X}\right]$ for any indecomposable $X$. 
However, even if all indecomposables are $d$-th CY objects with the same $d$, we do not know whether $\mathcal{A}$ is a Calabi-Yau category, although $F$ and $[d]$ coincide on objects. The examples we know have a positive answer to this question.

\section{Minimal Calabi-Yau objects}

The purpose of this section is to describe all the Calabi-Yau objects of a Homfinite Krull-Schmidt triangulated $k$-category with a Serre functor.

4.1. Let $\mathcal{A}$ be a Hom-finite Krull-Schmidt triangulated $k$-category. A $d$-th CY object $X$ is said to be minimal if any proper direct summand of $X$ is not a $d$-th CY object.

Lemma 4.1. Let $\mathcal{A}$ be a Hom-finite Krull-Schmidt triangulated k-category with a right Serre functor $F$. Then a non-zero object $X$ is a minimal d-th $C Y$ object if and only if the following are satisfied:

1. The indecomposable direct summands of $X$ can be ordered as $X=X_{1} \oplus \cdots \oplus X_{r}$ such that

$$
F\left(X_{1}\right) \cong X_{2}[d], F\left(X_{2}\right) \cong X_{3}[d], \cdots, F\left(X_{r-1}\right) \cong X_{r}[d], F\left(X_{r}\right) \cong X_{1}[d] .
$$

We call the cyclic order arising from this property a canonical order of $X$ (with respect to $F$ and $[d]$ ).

2. $X$ is multiplicity-free, i.e. its indecomposable direct summands are pairwise non-isomorphic.

Proof. In the following we often use that a non-zero object $X$ is a $d$-th CY object if and only if $F(X) \cong X[d]$.

Let $X=X_{1} \oplus \cdots \oplus X_{r}$ be a minimal $d$-th CY object, with each $X_{i}$ indecomposable and $r \geq 2$. Then $F\left(X_{1}\right) \oplus \cdots \oplus F\left(X_{r}\right) \cong X_{1}[d] \oplus \cdots \oplus X_{r}[d]$. Since $\mathcal{A}$ is KrullSchmidt, it follows that there exists a permutation $\sigma$ of $1, \cdots, r$, such that $F\left(X_{i}\right) \cong$ $X_{\sigma(i)}[d]$ for each $i$. Write $\sigma$ as a product of disjoint cyclic permutations. Since $X$ is minimal, it follows that $\sigma$ has to be a cyclic permutation of length $r$. By reordering the indecomposable direct summands of $X$, one may assume that $\sigma=(12 \cdots r)$. Thus, $X$ satisfies condition 1 .

Now, we consider a canonical order $X=X_{1} \oplus \cdots \oplus X_{r}$. If $X_{i} \cong X_{j}$ for some $1 \leq i<j \leq r$, then $F\left(X_{j-1}\right) \cong X_{j}[d] \cong X_{i}[d]$. It follows that $X_{i} \oplus \cdots \oplus X_{j-1}$ is already a $d$-th CY object, which contradicts the minimality of $X$. This proves that $X$ is multiplicity-free.

Conversely, assume that a multiplicity-free object $X=X_{1} \oplus \cdots \oplus X_{r}$ is in a canonical order. By (4.1) we have $F(X) \cong X[d]$, so $X$ is a $d$-th CY object. It remains to show the minimality. If not, then there exists a proper direct summand $X_{i_{1}} \oplus \cdots \oplus X_{i_{t}}$ of $X$ which is a minimal $d$-th CY object, so $1 \leq t<r$. By what we have proved above we may assume that this is a canonical order. Then

$$
F\left(X_{i_{1}}\right) \cong X_{i_{2}}[d], \cdots, F\left(X_{i_{t-1}}\right) \cong X_{i_{t}}[d], F\left(X_{i_{t}}\right) \cong X_{i_{1}}[d] .
$$

While $X=X_{1} \oplus \cdots \oplus X_{r}$ is also in a canonical order, it follows that (note that we work on indices modulo $r$, e.g., if $i_{1}=r$, then $i_{1}+1$ is understood to be 1 )

$$
F\left(X_{i_{1}}\right) \cong X_{i_{1}+1}[d], \cdots, F\left(X_{i_{t-1}}\right) \cong X_{i_{t-1}+1}[d], F\left(X_{i_{t}}\right) \cong X_{i_{t}+1}[d] .
$$


Since $X$ is multiplicity-free, it follows that (considering indices modulo $r$ )

$$
i_{2}=i_{1}+1, \cdots, i_{t}=i_{t-1}+1, i_{1}=i_{t}+1 ;
$$

hence $i_{1}=i_{1}+t$, which means $r \mid t$. This is impossible since $1 \leq t<r$.

4.2. Let $\mathcal{A}$ be a Hom-finite Krull-Schmidt triangulated $k$-category with Serre functor $F$. For each $d \in \mathbb{Z}$, consider the triangle-equivalence $G:=[-d] \circ F \cong F \circ[-d]$ : $\mathcal{A} \longrightarrow \mathcal{A}$. For each indecomposable $M \in \mathcal{A}$, denote by $o\left(G_{M}\right)$ the relative order of $G$ with respect to $M$. That is, $r:=o\left(G_{M}\right)$ is the minimal positive integer such that $G^{r}(M) \cong M$; otherwise $o\left(G_{M}\right)=\infty$. Denote by $\operatorname{Aut}(\mathcal{A})$ the group of the triangle-equivalences of $\mathcal{A}$ and by $\langle G\rangle$ the cyclic subgroup of $\operatorname{Aut}(\mathcal{A})$ generated by $G$. Then $\langle G\rangle$ acts naturally on $\operatorname{Ind}(\mathcal{A})$, the set of the isoclasses of indecomposables of $\mathcal{A}$. Denote by $\mathcal{O}_{M}$ the $G$-orbit of an indecomposable $M$. Then $\left|\mathcal{O}_{M}\right|=o\left(G_{M}\right)$. If $\left|\mathcal{O}_{M}\right|<\infty$, then the set $\mathcal{O}_{M}$ is a finite $G$-orbit.

Denote by $\operatorname{Fin} \mathcal{O}(\mathcal{A}, d)$ the set of all the finite $G$-orbits of $\operatorname{Ind}(\mathcal{A})$ and by $\operatorname{MinCY}(\mathcal{A}, d)$ the set of isoclasses of minimal $d$-th $\mathrm{CY}$ objects. We have the following

Theorem 4.2. Let $\mathcal{A}$ be a Hom-finite Krull-Schmidt triangulated $k$-category with Serre functor $F$. Then

(i) Every d-th $C Y$ object is a direct sum of finitely many minimal d-th $C Y$ objects.

(ii) With the notation above, for each $d \in \mathbb{Z}$ the map

$$
\mathcal{O}_{M} \mapsto \bigoplus_{X \in \mathcal{O}_{M}} X=M \oplus G(M) \oplus \cdots \oplus G^{o\left(G_{M}\right)-1}(M)
$$

gives a one-to-one correspondence between the sets $\operatorname{Fin} \mathcal{O}(\mathcal{A}, d)$ and $\operatorname{MinCY}(\mathcal{A}, d)$, where $G:=[-d] \circ F$.

Thus, a minimal d-th $C Y$ object is exactly the direct sum of all the objects in a finite $G$-orbit of $\operatorname{Ind}(\mathcal{A})$.

(iii) Non-isomorphic minimal d-th $C Y$ objects are disjoint, i.e., they have no isomorphic indecomposable direct summands.

Proof. Let $X$ be a $d$-th CY object. If $X$ is not minimal, then $X=Y \oplus Z$ with $Y \neq 0 \neq Z$ such that $F(Y) \oplus F(Z) \cong Y[d] \oplus Z[d]$ and $F(Y) \cong Y[d]$. Since $\mathcal{A}$ is Krull-Schmidt, it follows that $F(Z) \cong Z[d]$, i.e., $Z$ is also a $d$-th CY object. Then (i) follows by induction.

Thanks to Lemma 2.1, (4.1) becomes $X_{i}=G^{i-1}\left(X_{1}\right), 1 \leq i \leq r$. Then $(i i)$ is a reformulation of Lemma 4.1. Moreover (iii) follows from (ii).

Corollary 4.3. Let $A$ be a finite-dimensional self-injective algebra. Then $X$ is a minimal d-th $C Y$ module if and only if $X$ is of the form $X \cong \bigoplus_{0 \leq i \leq r-1} G^{i}(M)$, where $M$ is an indecomposable non-projective A-module with $r:=o\left(\bar{G}_{M}\right)<\infty$, and $G:=\Omega^{d+1} \circ \mathcal{N}$.

Proof. Note that in this case $[-d] \circ F=\Omega^{d+1} \circ \mathcal{N}$.

4.3. As an example, we describe all the Calabi-Yau objects in $D^{b}(k Q$-mod), the bounded derived category of $k Q$-mod, where $Q$ is a finite quiver without oriented cycles. 
Note that indecomposable objects of $D^{b}(k Q$-mod) are exactly stalk complexes of indecomposable $k Q$-modules. The category $D^{b}(k Q$-mod) has Serre functor $F=$ $[1] \circ \tau_{D}$, where $\tau_{D}$ is the Auslander-Reiten translation of $D^{b}(k Q$-mod). Recall that $\tau_{D}$ is given by

$$
\tau_{D}(M)= \begin{cases}\tau(M), & \text { if } M \text { is an indecomposable non-projective, } \\ I[-1], & \text { if } P \text { is an indecomposable projective, }\end{cases}
$$

where $I$ is the indecomposable injective with $\operatorname{soc}(I)=P / \operatorname{rad} P$ and $\tau$ is the Auslander-Reiten translation of $k Q-\bmod \left(\left[\right.\right.$ Hap1], p. 51). Note that $D^{b}(k Q-\bmod )$ is not a Calabi-Yau category except that $Q$ is the trivial quiver with one vertex and no arrows. However, the cluster category $\mathcal{C}_{k Q}$ introduced in [BMRRT, which is the orbit category of $D^{b}\left(k Q\right.$-mod) with respect to the functor $\tau_{D}^{-1} \circ[1]$, is a Calabi-Yau category of CY dimension 2 [BMRRT].

Let $M$ be a minimal Calabi-Yau object of $D^{b}(k Q$-mod) of CY dimension $d$ (in this case $o\left([1]_{M}\right)=\infty$, hence $d$ is unique). By shifts we may assume that $M$ is a $k Q$ module. By $F(M)=\tau_{D}(M[1])=\tau_{D}(M)[1]=M[d]$ we see $d=1$ or 0 . Note that $k Q$ admits an indecomposable projective-injective module if and only if $Q$ is of type $A_{n}$ with the linear orientation. However, in this case the unique indecomposable projective-injective module $P=I$ does not satisfy the relation $\operatorname{soc}(I)=P / \operatorname{rad} P$. It follows that $d \neq 0$. Thus $d=1$ and $\tau(M)=M$. Consequently, $Q$ is an affine quiver and $M$ is a $\tau$-periodic (regular) module of period 1. All such modules are well known by the classification of representations of affine quivers (see Dlab-Ringel [DR]). Thus we have

Proposition 4.4. $D^{b}(k Q-m o d)$ admits a Calabi-Yau object $M$ if and only if $Q$ is an affine quiver. In this case, $M$ is minimal if and only if $M$ is indecomposable in a homogeneous tube of the Auslander-Reiten quiver of $k Q$-mod, or $M$ is the direct sum of all the indecomposables of the same quasi-length in a non-homogeneous tube of the Auslander-Reiten quiver of $k Q$-mod, up to shifts. Moreover, all such $M$ 's have $C Y$ dimension 1.

\section{Calabi-Yau modules of Self-injective Nakayama algebras}

The purpose of this section is to classify all the $d$-th CY modules of self-injective Nakayama algebras $\Lambda(n, t), n \geq 1, t \geq 2$, where $d$ is any given integer. By Theorem $4.2(i)$ it suffices to consider the minimal $d$-th CY $\Lambda$-modules. By Corollary 4.3 this reduces to computing the relative order $o\left(G_{M}\right)$ of $G:=\Omega^{d+1} \circ \mathcal{N}$ with respect to any indecomposable $\Lambda(n, t)$-module $M=S_{i}^{l}, 1 \leq l \leq t-1$, for any integer $d$.

5.1. Recall that $\Lambda(n, t)$ is the quotient of the path algebra of the cyclic quiver with $n$ vertices by the truncated ideal $J^{t}$, where $J$ is the two-sided ideal generated by the arrows. From now on we write $\Lambda$ instead of $\Lambda(n, t)$.

We keep the notation introduced in $\S 2.5$. Note that the indecomposable module $S_{i}^{l}(i \in \mathbb{Z} / n \mathbb{Z}, \quad 1 \leq l \leq t-1)$ has a natural $k$-basis, consisting of all the paths of quiver $\mathbb{Z}_{n}$ starting at the vertex $i+l-t$ and of lengths at least $t-l$ :

$$
\gamma_{i+l-t}^{t-l}, \gamma_{i+l-t}^{t-l+1}, \cdots, \gamma_{i+l-t}^{t-1} .
$$

For $1 \leq l \leq t-1$ denote by $\sigma_{i}^{l}: S_{i}^{l} \longrightarrow S_{i-1}^{l+1}$ the inclusion by embedding the basis above; for $2 \leq l \leq t$ denote by $p_{i}^{l}: S_{i}^{l} \longrightarrow S_{i}^{l-1}$ the $\Lambda$-epimorphism given by the 
right multiplication by arrow $a_{i+l-t-1}$. These $\sigma_{i}^{l}$,s and $p_{i}^{l}$, s are all the irreducible maps of $\Lambda$-mod, up to scalars.

We need the explicit actions of functors $\mathcal{N}$ and $\Omega^{-(d+1)}$ of $\Lambda$-mod. By the exact sequences $0 \longrightarrow S_{i}^{l} \longrightarrow I(i+l-1) \longrightarrow S_{i+l-t}^{t-l} \longrightarrow 0$ (with the canonical maps), via the basis above one has the actions of functor $\Omega^{-1}$ for $i \in \mathbb{Z} / n \mathbb{Z}, \quad 1 \leq l \leq t-1$,

$$
\Omega^{-1}\left(S_{i}^{l}\right)=S_{i+l-t}^{t-l}, \quad \Omega^{-1}\left(\sigma_{i}^{l}\right)=p_{i+l-t}^{t-l}, \quad \Omega^{-1}\left(p_{i}^{l}\right)=\sigma_{i+l-t}^{t-l} .
$$

By induction one has in $\Lambda$-mod for any integer $m$ (even negative):

$$
\Omega^{-(2 m-1)}\left(S_{i}^{l}\right)=S_{i+l-m t}^{t-l}, \quad \Omega^{-2 m}\left(S_{i}^{l}\right)=S_{i-m t}^{l}
$$

and

$$
\Omega^{-(2 m-1)}\left(\sigma_{i}^{l}\right)=p_{i+l-m t}^{t-l}, \quad \Omega^{-(2 m-1)}\left(p_{i}^{l}\right)=\sigma_{i+l-m t}^{t-l}
$$

and

$$
\Omega^{-2 m}\left(\sigma_{i}^{l}\right)=\sigma_{i-m t}^{l}, \quad \Omega^{-2 m}\left(p_{i}^{l}\right)=p_{i-m t}^{l} .
$$

In particular, we have

$$
o([1])= \begin{cases}n, & t=2 \\ 2 m, & t \geq 3,\end{cases}
$$

where $m$ is the minimal positive integer such that $n \mid m t$.

5.2. Again using the natural basis of $S_{i}^{l}$ one has the following commutative diagrams in $\Lambda$-mod:
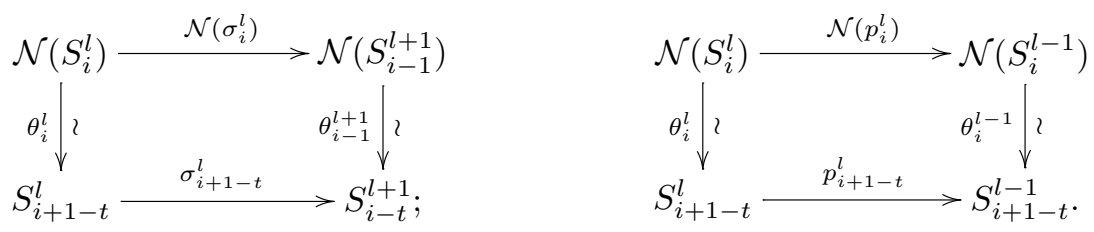

We justify the commutative diagrams above. Note that for any finite quiver $Q$ the bimodule structure of $D(k Q)$ is given by (using the dual basis)

$$
p^{*} a=\left\{\begin{array}{ll}
b^{*}, & \text { if } a b=p, \\
0, & \text { otherwise }
\end{array} \quad \text { and } \quad a p^{*}= \begin{cases}b^{*}, & \text { if } b a=p, \\
0, & \text { otherwise }\end{cases}\right.
$$

for any paths $p$ and $a$. Note that $N\left(S_{i}^{l}\right)=D(\Lambda) \otimes_{\Lambda} S_{i}^{l}$ is spanned by $\left(\gamma_{j}^{l^{\prime}}\right)^{*} \otimes_{\Lambda} \gamma_{i+l-t}^{t-u}$, where $j \in \mathbb{Z} / n \mathbb{Z}, 1 \leq l^{\prime} \leq t-1,1 \leq u \leq l$. By $\left(\gamma_{j}^{l^{\prime}}\right)^{*} \otimes_{\Lambda} \gamma_{i+l-t}^{t-u}=\left(\gamma_{j}^{l^{\prime}}\right)^{*} \gamma_{i+l-t}^{t-u} \otimes_{\Lambda}$ $e_{i+l-t}$ we see that if $\left(\gamma_{j}^{l^{\prime}}\right)^{*} \otimes_{\Lambda} \gamma_{i+l-t}^{t-u} \neq 0$, then $j=i+l-l^{\prime}-u$. In this case we have

$$
\left(\gamma_{i+l-l^{\prime}-u}^{l^{\prime}}\right)^{*} \otimes_{\Lambda} \gamma_{i+l-t}^{t-u}=\left(\gamma_{i+l-l^{\prime}-u}^{l^{\prime}}\right)^{*} \gamma_{i+l-t}^{t-u} \otimes_{\Lambda} e_{i+l-t}=\left(\gamma_{i+l-\left(l^{\prime}+u\right)}^{l^{\prime}+u-t}\right)^{*} \otimes_{\Lambda} e_{i+l-t} .
$$

This makes sense only if $l^{\prime}+u \geq t$. So we have a basis of $N\left(S_{i}^{l}\right)$ :

$$
\left(\gamma_{i+l-t-v}^{v}\right)^{*} \otimes_{\Lambda} e_{i+l-t}, \quad 0 \leq v \leq l-1 .
$$

Using the natural basis of $S_{i+1-t}^{l}$ given in $\S 5.1$ we have a $\Lambda$-isomorphism $\theta_{i}^{l}$ : $\mathcal{N}\left(S_{i}^{l}\right) \longrightarrow S_{i+1-t}^{l}$ for any $i \in \mathbb{Z} / n \mathbb{Z}$ and $1 \leq l \leq t-1$ :

$$
\theta_{i}^{l}:\left(\gamma_{i+l-t-v}^{v}\right)^{*} \otimes_{\Lambda} e_{i+l-t} \mapsto \gamma_{i+1+l-2 t}^{t-(v+1)}, \quad 0 \leq v \leq l-1 .
$$


(One checks that this is indeed a left $\Lambda$-map.) Note that $N\left(\sigma_{i}^{l}\right): \mathcal{N}\left(S_{i}^{l}\right) \longrightarrow$ $\mathcal{N}\left(S_{i-1}^{l+1}\right)$ is a natural embedding given by

$$
\left(\gamma_{i+l-t-v}^{v}\right)^{*} \otimes_{\Lambda} e_{i+l-t} \mapsto\left(\gamma_{i+l-t-v}^{v}\right)^{*} \otimes_{\Lambda} e_{i+l-t}, \quad 0 \leq v \leq l-1,
$$

and that $N\left(p_{i}^{l}\right): \mathcal{N}\left(S_{i}^{l}\right) \longrightarrow \mathcal{N}\left(S_{i}^{l-1}\right)$ is a $\Lambda$-epimorphism given by

$$
\left(\gamma_{i+l-t-v}^{v}\right)^{*} \otimes_{\Lambda} e_{i+l-t} \mapsto\left(\gamma_{i+l-t-v}^{v-1}\right)^{*} \otimes_{\Lambda} e_{i+l-1-t}, \quad 0 \leq v \leq l-1,
$$

where $\gamma_{i+l-1-t}^{-1}$ is understood to be 0 . Then one easily checks the following:

$$
\sigma_{i+1-t}^{l} \circ \theta_{i}^{l}=\theta_{i-1}^{l+1} \circ \mathcal{N}\left(\sigma_{i}^{l}\right) ; \quad p_{i+1-t}^{l} \circ \theta_{i}^{l}=\theta_{i}^{l-1} \circ \mathcal{N}\left(p_{i}^{l}\right) .
$$

This justifies the commutative diagrams.

Since all these $\theta_{i}^{l}$ depend only on $i$ and $l$, which means that they do not depend on whatever the maps $\sigma_{i}^{l}$ or $p_{i}^{l}$ are (this is important for the bi-naturality of a CalabiYau category), it follows, without loss of the generality, that we can specialize these maps to identities. Thus we have

$$
\mathcal{N}\left(S_{i}^{l}\right)=S_{i+1-t}^{l}, \quad \mathcal{N}\left(\sigma_{i}^{l}\right)=\sigma_{i+1-t}^{l}, \quad \mathcal{N}\left(p_{i}^{l}\right)=p_{i+1-t}^{l} .
$$

5.3. By $(* * *)$ we have $o([1])<\infty$. It follows, without loss of generality, that we can assume $d \geq 0$. For convenience, set $d(t):=1+\frac{(d-1) t}{2} \in \frac{1}{2} \mathbb{Z}$, whenever $d$ is even or odd; denote by $N=N(d, n, t)$ the minimal positive integer such that

$$
\begin{cases}n \mid N d(t), & \text { if }(d-1) t \text { is even; } \\ n \mid N(2 d(t)), & \text { if }(d-1) t \text { is odd. }\end{cases}
$$

(When $2 d(t)$ is odd, we will write it together in the following.)

By (5.1) we have $\Omega^{(2 m-1)}\left(S_{i}^{l}\right)=S_{i+l+(m-1) t}^{t-l}$ and $\Omega^{2 m}\left(S_{i}^{l}\right)=S_{i+m t}^{l}$. Hence by (5.2) we have (remember $G:=\Omega^{d+1} \circ \mathcal{N}$ and $d \geq 0$ )

$$
G\left(S_{i}^{l}\right)=S_{i+1+(m-1) t}^{l}, \text { if } d=2 m-1
$$

and

$$
G\left(S_{i}^{l}\right)=S_{i+l+1+(m-1) t}^{t-l}, \text { if } d=2 m .
$$

Thus, by induction we have

$$
G^{m^{\prime}}\left(S_{i}^{l}\right)=S_{i+m^{\prime}(1+(m-1) t)}^{l}=S_{i+m^{\prime} d(t)}^{l}, \text { if } d=2 m-1 .
$$

Also,

$$
G^{2 m^{\prime}}\left(S_{i}^{l}\right)=S_{i+m^{\prime}(2+(2 m-1) t)}^{l}=S_{i+m^{\prime}(2 d(t))}^{l}, \text { if } d=2 m
$$

and

$$
G^{2 m^{\prime}+1}\left(S_{i}^{l}\right)=S_{i+\left(m^{\prime}+1\right)(2 d(t))+l-1-m t}^{t-l}, \text { if } d=2 m,
$$

for $m^{\prime} \geq 0$. 
5.4. If $d=2 m-1 \geq 1$, then by (5.4) we see that $o\left(G_{S_{i}^{l}}\right)=N$, where $N$ is as given in (5.3), i.e., $N$ is the minimal positive integer such that $n \mid N(1+(m-1) t)$. It follows from Corollary 4.3 and (5.4) that we have

Lemma 5.1. Let $d=2 m-1 \geq 1$. Then $M$ is a minimal d-th $C Y \Lambda$-module if and only if $M$ is isomorphic to one of the following:

$$
S_{i}^{l} \oplus S_{i+d(t)}^{l} \oplus S_{i+2 d(t)}^{l} \oplus \cdots \oplus S_{i+(N-1) d(t)}^{l}, \quad 1 \leq l \leq t-1, \quad i \in \mathbb{Z} / n \mathbb{Z} .
$$

In particular, all the minimal $d$-th $C Y$ modules have the same number $N=$ $N(d, n, t)$ of indecomposable direct summands.

5.5. If $d=2 m \geq 0$ and $t$ is odd, then by (5.6) we see $G^{2 m^{\prime}+1}\left(S_{i}^{l}\right) \neq S_{i}^{l}$ for any $i, l$ (since $t-l \neq l$ ). Note that in this case $(d-1) t$ is odd. It follows from (5.5) that $o\left(G_{S_{i}^{l}}\right)=2 N$, where $N$ is as given in (5.3), i.e., $N$ is the minimal positive integer such that $n \mid N(2 d(t))$. It follows from Corollary 4.3, (5.5) and (5.6) that we have

Lemma 5.2. Let $t \geq 3$ be an odd integer and $d=2 m \geq 0$. Then $M$ is a minimal $d$-th $C Y \Lambda$-module if and only if $M$ is isomorphic to one of the following:

$$
S_{i}^{l} \oplus S_{i+l^{\prime}+2 d(t)}^{t-l} \oplus S_{i+2 d(t)}^{l} \oplus S_{i+l^{\prime}+4 d(t)}^{t-l} \oplus \cdots \oplus S_{i+2 d(t)(N-1)}^{l} \oplus S_{i+l^{\prime}+2 d(t) N}^{t-l}
$$

where $l^{\prime}:=l-1-m t, \quad 1 \leq l \leq t-1$ and $i \in \mathbb{Z} / n \mathbb{Z}$.

In particular, any minimal $d$-th $C Y$ module has $2 N=2 N(d, n, t)$ indecomposable direct summands.

5.6. Let $d=2 m \geq 0$ and $t=2 s$. Then $d(t)=1+(2 m-1) s \in \mathbb{Z}$.

First, we consider $o\left(G_{S_{i}^{s}}\right)$. In this case (5.5) and (5.6) can be written in a unified way:

$$
G^{m^{\prime}}\left(S_{i}^{s}\right)=S_{i+m^{\prime} d(t)}^{s}, m^{\prime} \geq 0, \text { if } d=2 m, t=2 s .
$$

So we have $o\left(G_{S_{i}^{s}}\right)=N$, where $N$ is as given in (5.3), i.e., $N$ is the minimal positive integer such that $n \mid N(1+(2 m-1) s)$.

Now, we consider $o\left(G_{S_{i}^{l}}\right)$ with $l \neq s, 1 \leq l \leq t-1$. In this case (5.5) and (5.6) are written respectively as

$$
G^{2 m^{\prime}}\left(S_{i}^{l}\right)=S_{i+2 m^{\prime} d(t)}^{l} \text {, if } d=2 m, t=2 s, l \neq s,
$$

and

$$
G^{2 m^{\prime}+1}\left(S_{i}^{l}\right)=S_{i+\left(2 m^{\prime}+1\right) d(t)+l-s}^{t-l}, \text { if } d=2 m, t=2 s, l \neq s
$$

for $m^{\prime} \geq 0$. Since $l \neq t-l$ for $l \neq s$, it follows that $G^{2 m^{\prime}+1}\left(S_{i}^{l}\right) \neq S_{i}^{l}$. So by (5.10) we see $o\left(G_{S_{i}^{l}}\right)=2 N^{\prime}$, where $N^{\prime}$ is the minimal positive integer such that $n \mid 2 N^{\prime} d(t)$. In order to determine $N^{\prime}$, we divided it into two cases.

Case 1. If $N=N(d, n, t)=N(2 m, n, 2 s)$ is even, then $o\left(G_{S_{i}^{l}}\right)=N$ for any $1 \leq$ $l \leq t-1$. It follows from Corollary 4.3, (5.9), (5.10), and (5.11) that we have the following (note that in this case (5.9) is exactly (5.10) together with (5.11), by taking $l=s$ ). 
Lemma 5.3. Let $t=2 s$ and $d=2 m \geq 0$. Assume that $N=N(d, n, t)$ is even. Then $M$ is a minimal d-th $C Y \Lambda$-module if and only if $M$ is isomorphic to one of the following:

$S_{i}^{l} \oplus S_{i+d(t)+l-s}^{t-l} \oplus S_{i+2 d(t)}^{l} \oplus S_{i+3 d(t)+l-s}^{t-l} \oplus \cdots \oplus S_{i+(N-2) d(t)}^{l} \oplus S_{i+(N-1) d(t)+l-s}^{t-l}$, where $1 \leq l \leq t-1, i \in \mathbb{Z} / n \mathbb{Z}$.

In particular, all the minimal d-th $C Y$ modules have the same number $N$ of indecomposable direct summands.

It remains to deal with

Case 2. Let $N=N(d, n, t)=N(2 m, n, 2 s)$ be odd. Since by definition $N$ is the minimal positive integer such that $n \mid N d(t)$, it follows that $N<o\left(G_{S_{i}^{l}}\right)=2 N^{\prime} \leq$ $2 N$. It is easy to see $N^{\prime}=N$; otherwise $1 \leq 2 N^{\prime}-N \leq N-1$ and $n \mid\left(2 N^{\prime}-N\right) d(t)$, which contradicts the minimality of $N$. It follows from Corollary 4.3, (5.9), (5.10), and (5.11) that we have

Lemma 5.4. Let $t=2 s$ and $d=2 m \geq 0$. Assume that $N=N(d, n, t)$ is odd. Then $M$ is a minimal d-th $C Y \Lambda$-module if and only if $M$ is isomorphic to one of the following:

$$
S_{i}^{s} \oplus S_{i+d(t)}^{s} \oplus S_{i+2 d(t)}^{s} \oplus \cdots \oplus S_{i+(N-1) d(t)}^{s},
$$

where $i \in \mathbb{Z}_{n}$, and

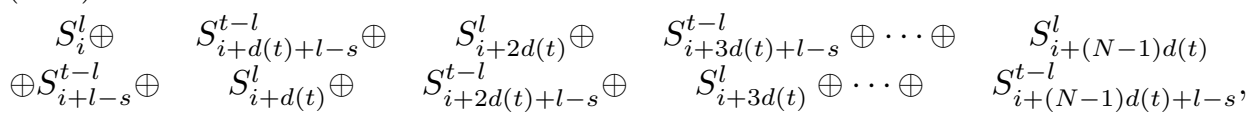
where $l \neq s, \quad 1 \leq l \leq t-1$ and $i \in \mathbb{Z}_{n}$.

In particular, all the minimal d-th $C Y$ modules have either $N$ or $2 N$ indecomposable direct summands.

5.7. By Lemmas 5.1-5.4 all the minimal $d$-th CY modules of self-injective Nakayama algebras have been classified, where $d$ is any given integer. The main result of this section is as follows.

Theorem 5.5. For any $n \geq 1, t \geq 2, d \geq 0$, let $N=N(d, n, t)$ be as in (5.3). Then $M$ is a minimal d-th $C Y \Lambda$-module if and only if $M$ is isomorphic to one of the following:

(i) The modules in (5.7), when $d=2 m-1$.

(ii) The modules in (5.8), when $d=2 m$ and $t$ is odd.

(iii) The modules in (5.12), when $d=2 m, t=2 s$, and $N(d, n, t)$ is even.

(iv) The modules in (5.13) - (5.14), when $d=2 m, t=2 s$, and $N(d, n, t)$ is odd.

In particular, any minimal d-th $C Y \Lambda$-module has either $N$ or $2 N$ indecomposable direct summands, and

$$
\min \{d \geq 0 \mid N=c(M) \text { or } 2 N=c(M)\} \leq \mathrm{CYdim}(M)<o\left([1]_{M}\right) \leq 2 n
$$

for any minimal Calabi-Yau $\Lambda$-modules $M$, where $c(M)$ is the number of indecomposable direct summands of $M$.

Proof. By Lemmas 5.1-5.4 the CY dimension $d$ of any minimal Calabi-Yau module $M$ satisfies $N(d, n, t)=c(M)$ or $2 N(d, n, t)=c(M)$. 
Remark 5.6. The modules in (5.7) - (5.8) and (5.12) - (5.14) have overlaps. This is because $d$ is not uniquely determined by a minimal $d$-th CY module. A general formula of the CY dimensions of the minimal Calabi-Yau modules seems to be difficult to obtain.

Note that the inequality on the left-hand side in (5.15) cannot be an equality in general. For example, take $n=2, t=4, m=2, d=2 m-1=3$. Then $d(t)=5$, $N=N(3,2,4)=2$, and $S_{i}^{l} \oplus S_{i+1}^{l}, 1 \leq l \leq 3$, are all the minimal 3-rd CY modules of $C Y$ dimension 0 if $l=2$, and 1 if $l=1,3$. However, the left-hand side in (5.15) is 0 since $N(0,2,4)=2$.

\section{Self-injective Nakayama algebras With INDECOMPOSABLE CALABI-YAU MODUlES}

In this section we determine all the self-injective Nakayama algebras $\Lambda=\Lambda(n, t)$, $n \geq 1, t \geq 2$, which admit indecomposable Calabi-Yau modules.

Note that Erdmann and Skowroński have proved in $\S 2$ of [ES] that self-injective algebras $A$ such that $A$-mod is Calabi-Yau of CY dimension 0 (resp. 1) are the algebras Morita equivalent to $\Lambda(n, 2)$ for some $n \geq 1$ (resp. $\Lambda(1, t)$ for some $t \geq 3$ ). So we assume that $t \geq 3$.

Theorem 6.1. Let $t \geq 3$. Then $\Lambda$ has an indecomposable Calabi-Yau module if and only if $n$ and $t$ satisfy one of the following conditions:

(i) g.c.d. $(n, t)=1$. This is exactly the case where $\Lambda$-mod is a Calabi-Yau category. In this case we have $\mathrm{CY} \operatorname{dim}(\Lambda)=2 m-1$, where $m$ is the minimal positive integer such that $n \mid(m-1) t+1$.

(ii) g.c.d. $(n, t) \neq 1, t=2 s$, and g.c.d. $(n, s)=1$. This is exactly the case where $\Lambda$-mod is not a Calabi-Yau category but admits indecomposable Calabi-Yau modules.

In this case, we have g.c.d. $(n, t)=2$ and

(a) $S_{i}^{s}, i \in \mathbb{Z} / n \mathbb{Z}$, are all the indecomposable Calabi-Yau modules.

(b) $\quad S_{i}^{l} \oplus S_{i+l-s}^{t-l}, \quad 1 \leq l \leq s-1, i \in \mathbb{Z} / n \mathbb{Z}$, are all the decomposable minimal 2m-th $C Y$ modules, where $m$ is the minimal non-negative integer such that $n \mid(2 m-1) s+1$.

(c) All of these modules in (a) and (b) have the same $C Y$ dimension $2 m$.

Remark. Bialkowski and Skowroński [BS] have classified representation-finite selfinjective algebras whose stable categories are Calabi-Yau. This includes assertion (i) of Theorem 6.1.

Proof. If $\Lambda$ has an indecomposable $d$-th CY module $S_{i}^{l}$, then $\mathcal{N}\left(S_{i}^{l}\right) \cong \Omega^{-(d+1)}\left(S_{i}^{l}\right)$. By (5.2) and (5.1) we have

$$
1-t \equiv-m t(\bmod n), \text { if } d+1=2 m
$$

or

$$
t=t-l, 1-t \equiv l-m t(\bmod n), \text { if } d+1=2 m-1 .
$$

In the first case we get g.c.d. $(n, t)=1$. In the second case we get $t=2 s, l=s$ and g.c.d. $(n, s)=1$. Excluding the overlap situations we conclude that either g.c.d. $(n, t)=1$ or g.c.d. $(n, t) \neq 1, t=2 s$, g.c.d. $(n, s)=1$.

Assume that g.c.d. $(n, t)=1$. Then there exists an integer $m$ such that $n \mid$ $(m-1) t+1$. We choose a positive (otherwise, add $n(-m+2) t$ ) and minimal $m$. 
Set $d:=2 m-1$. Then the same computation shows that every indecomposable module is a $d$-th CY module. We claim that $\Lambda$-mod is a Calabi-Yau category. For this, it remains to show $\mathcal{N}(f)=\Omega^{-(d+1)}(f)=\Omega^{-2 m}(f)$ for any morphism $f$ between indecomposables of $\Lambda$-mod. Since $n \mid(m-1) t+1$, it follows from (**) in $\S 5$ that

$$
\mathcal{N}\left(\sigma_{i}^{l}\right)=\Omega^{-2 m}\left(\sigma_{i}^{l}\right), \quad \mathcal{N}\left(p_{i}^{l}\right)=\Omega^{-2 m}\left(p_{i}^{l}\right) .
$$

Since $\Lambda$ is representation-finite, it follows that $f$ is a $k$-combination of compositions of irreducible maps $\sigma_{i}^{l}$ and $p_{i}^{l}$; hence $\mathcal{N}(f)=\Omega^{-2 m}(f)$ by (6.3). We stress here that this argument relies on the fact that all the isomorphisms $\theta_{i}^{l}$ in $\S 5.2$ depend only on $i$ and $l$, which means that they do not depend on whatever the maps $\sigma_{i}^{l}$ or $p_{i}^{l}$ are. Otherwise we cannot take them as identities, and then we cannot get the naturality for the Calabi-Yau category of this case.

This proves the claim; hence $\Lambda$-mod is a Calabi-Yau category of CY dimension $D$, with $0 \leq D \leq d=2 m-1$. We claim $D=d$. In fact, since every indecomposable module is a $D$-th CY module, and since we have $l$ such that $t \neq t-l$, it follows from (6.1) and (6.2) that $D=2 m^{\prime}-1$ with $n \mid\left(m^{\prime}-1\right) t+1$, hence $D=d$, by the minimality of $m$.

The argument above also proves that if $\Lambda$-mod is a Calabi-Yau category, then g.c.d. $(n, t)=1$.

Assume that g.c.d. $(n, t) \neq 1, t=2 s$, and g.c.d. $(n, s)=1$. Then there exists an integer $m^{\prime}$ such that $n \mid\left(m^{\prime}-1\right) s+1$. We choose a positive $m^{\prime}$. Since g.c.d. $(n, t) \neq 1$, it follows that $m^{\prime}$ is even, say $m^{\prime}=2 m$ with $m \geq 1$. Let $m$ be the minimal positive integer such that $n \mid(2 m-1) s+1$, and set $d:=2 m$. Then the same computation shows that $S_{i}^{s}, i \in \mathbb{Z} / n \mathbb{Z}$, are all the indecomposable Calabi-Yau modules. This proves $(a)$.

By applying Lemma 5.4 to $d$ given above (note that the corresponding $N=$ $N(n, d, t)=1$ in this case), we know that $S_{i}^{l} \oplus S_{i+l-s}^{t-l}, 1 \leq l \leq t-1, l \neq s, i \in$ $\mathbb{Z} / n \mathbb{Z}$, are all the decomposable minimal $2 m$-th $\mathrm{CY}$ modules. By symmetry one can consider $1 \leq l \leq s-1$ : if $l>s$ then one can replace $l$ by $t-l$, since $i=(i+l-s)+(t-l)-s$. This proves $(b)$.

It remains to prove $(c)$. Let $\mathrm{CY} \operatorname{dim}\left(S_{i}^{s}\right)=d^{\prime}$. Since g.c.d. $(n, t) \neq 1$, it follows that $d^{\prime}$ has to be an even integer $2 m^{\prime} \geq 0$ with $n \mid\left(2 m^{\prime}-1\right) s+1$. it follows that $d^{\prime}=d$, by the minimality of $m$. Let $\operatorname{CYdim}\left(S_{i}^{l} \oplus S_{i+l-s}^{t-l}\right)=d^{\prime}$. Then we have $\mathcal{N}\left(S_{i}^{l}\right)=\Omega^{-\left(d^{\prime}+1\right)}\left(S_{i+l-s}^{t-l}\right)$. Since $l \neq s$ it follows from (5.2) and (5.1) that $d^{\prime}$ has to be $2 m^{\prime} \geq 0$ with $n \mid\left(2 m^{\prime}-1\right) s+1$. Again by the minimality of $m$ we have $d^{\prime}=d$. This completes the proof.

Remark 6.2. (i) CYdim $(X)$ usually differs from $C Y \operatorname{dim}(\mathcal{A})$ in a Calabi-Yau category $\mathcal{A}$.

For example, if $n=3, t=4$, then $o([1])=6, \mathrm{CY} \operatorname{dim}(\Lambda)=5$, while $\mathrm{CY} \operatorname{dim}\left(S_{i}^{2}\right)=$ 2 and $o\left([1]_{S_{i}^{2}}\right)=3, \forall i \in \mathbb{Z} / 3 \mathbb{Z}$.

However, for $t \geq 3$ and g.c.d. $(n, t)=1$, if $X$ is indecomposable and $\operatorname{CYdim}(X)$ is odd, then $\mathrm{CY} \operatorname{dim}(X)=\mathrm{CY} \operatorname{dim}(\Lambda)$. In fact, since $o([1])<\infty$ it follows that $\operatorname{CYdim}(X)=2 m^{\prime}-1 \geq 1$, and $n \mid 1+\left(m^{\prime}-1\right) t$. By Theorem 6.1(i) CYdim $(\Lambda)=$ $2 m-1$, where $m$ is the minimal positive integer such that $n \mid 1+(m-1) t$. It follows that $m^{\prime} \geq m$, hence $C Y \operatorname{dim}(X) \geq \operatorname{CY} \operatorname{dim}(\Lambda)$. On the other hand we have $\mathrm{CY} \operatorname{dim}(X) \leq \mathrm{CY} \operatorname{dim}(\Lambda)$ by definition. 
(ii) Consider the algebra $A(t):=k A_{\infty}^{\infty} / J^{t}$, where $A_{\infty}^{\infty}$ is the infinite quiver

$$
\cdots \longrightarrow \bullet \longrightarrow \bullet \longrightarrow \bullet \longrightarrow \bullet \longrightarrow \bullet \longrightarrow \cdots \text {. }
$$

Then $A(t)$-mod has a Serre functor, and there is a natural covering functor $A(t) \underline{\text { mod }}$ $\longrightarrow \Lambda(n, t)$-mod $(\mathrm{Gab}, 2.8)$. But one can prove that in any case $A(t)$-mod is not a Calabi-Yau category.

\section{ACKNOWLEDGEMENTS}

This work was done during a visit of the second-named author at Université de Montpellier 2, supported by the CNRS of France. He thanks the first-named author and his group for their warm hospitality, the Département de Mathématiques of Université de Montpellier 2 for their working facilities, and the CNRS for their support. We thank Bernhard Keller for helpful conversations.

\section{REFERENCES}

[A] C. Amiot, On the structure of triangulated category with finitely many indecomposables. Available at arxiv: CT/0612141.

[AR] M. Auslander, and I. Reiten, Representation theory of Artin algebras III, Commun. in Algebra 3(1975), 239-294. MR0379599(52:504)

[ARS] M. Auslander, I. Reiten, and Smalø, Representation Theory of Artin Algebras, Cambridge Studies in Adv. Math. 36, Cambridge Univ. Press, 1995. MR.1314422 (96c:16015)

[BS] J. Bialkowski, and A. Skowroński, Calabi-Yau stable module categories of finite types. Colloq. Math. 109(2007), no. 2, 257-259. MR2318522 (2008f:16026)

[B] R. Bocklandt, Graded Calabi Yau algebras of dimension 3, with an appendix "The signs of Serre functor" by M. Van den Bergh. J. Pure Appl. Algebra 212(2008), no. 1, 14-32. MR2355031

[BK] A. Bondal, and M. Kapranov, Representable functors, Serre functors, and mutations, Math. USSR Izv. 35(1990), 519-541. MR1039961 (91b:14013)

[BMRRT] A. B. Buan, R. Marsh, M. Reineke, I. Reiten, and G. Todorov, Tilting theory and cluster combinatorics, Adv. Math. 204(2)(2006), 572-618. MR2249625(2007f:16033)

[Ch] X. W. Chen, Generalized Serre duality. Available at arXiv: RT/0610258.

[Co] K. Costello, Topological conformal field theories and Calabi-Yau categories. Adv. Math 210(2007), 165-214. MR2298823 (2008f:14071)

[DR] V. Dlab, and C. M. Ringel, Indecomposable representations of graphs and algebras, Memoirs Amer. Math. Soc. 173(1976). MR0447344 (56:5657)

[ES] K. Erdmann, and A. Skowroński, The stable Calabi-Yau dimension of tame symmetric algebras, J. Math. Soc. Japan 58(1)(2006), 97-128. MR2204567(2007b:16037)

[Gab] P. Gabriel, The universal cover of representation-finite algebras, Lecture Notes in Math. 903, Springer-Verlag, 1981, 68-105. MR654725 (83f:16036)

[GR] P. Gabriel, and C. Riedtmann, Group representations without groups, Comment. Math. Helv. 54(1979), 240-287. MR.535058 (80k:16040)

[Gin1] V. Ginzburg, Lectures on noncommutative gemotry. Available at arXiv: AG/0506603.

[Gin2] V. Ginzburg, Calabi-Yau algebras. Available at arXiv: AG/0612139.

[Hap1] D. Happel, Trianglated categories in representation theory of finite dimensional algebras, London Math. Soc. Lecture Notes Ser. 119, Cambridge Uni. Press, 1988. MR.935124 (89e:16035)

[Hap2] D. Happel, Auslander-Reiten triangles in derived categories of finite-dimensional algebras, Proc. Amer. Math. Soc. 112(3)(1991), 641-648. MR1045137 (91m:16015)

[Har] R. Hartshorne, Residue and duality, Lecture Notes in Math. 20, Springer-Verlag, 1966. MR.0222093 (36:5145)

[IR] O. Iyama, and I. Reiten, Fomin-Zelevinsky mutation and tilting modules over CalabiYau algebras. Amer. J. Math. 130(2008), no. 4, 1087-1149. MR2427009

[J] G. J. Janusz, Indecomposable modules for finite groups, Ann. Math. 89(1969), 209-241. MR 0244307 (39:5622) 
[Ke] B. Keller, On triangulated orbit categories, Documenta Math. 10(2005), 551-581. MR2184464 (2007c:18006)

[KR1] B. Keller, and I. Reiten, Cluster-tilted algebras are Gorenstein and stably Calabi-Yau. Adv. Math 211(2007), no. 1, 123-151. MR2313531 (2008b:18018)

[KR2] B. Keller, and I. Reiten, Acyclic Calabi-Yau categories are cluster categories. With an appendix by M. Van den Bergh. Compos. Math. 144(5)(2008), 1332-1348. MR2457529

[Ko] M. Kontsevich, Triangulated categories and geometry, Course at the École Normale Supérieure, Paris, Notes taken by J. Bellä̈che, J. F. Dat, I. Marin, G. Racinet, and H. Randriambololona, 1998.

[N] A. Neeman, Triangulated categories, Annals of Math. Studies, vol. 148, Princeton University Press, 2001. MR 1812507 (2001k:18010)

[RV] I. Reiten, and M. Van den Bergh, Noether hereditary abelian categories satisfying Serre functor, J. Amer. Math. Soc. 15(2)(2002), 295-366 (electronic). MR.1887637 (2003a:18011)

[R] C. M. Ringel, Tame algebras and integral quadratic forms, Lecture Notes in Math. 1099, Springer-Verlag, 1984. MR774589 (87f:16027)

[V] J. L. Verdier, Catégories dérivées, Lecture Notes in Math. 569, Springer-Verlag, 1977, 262-311. MR 0463174 (57:3132)

[XZ] J. Xiao, and B. Zhu, Locally finite triangulated categories, J. Algebra 290(2)(2005), 473-490. MR2153264 (2006c:18005)

Institut de Mathématiques et de Modélisation de Montpellier-I3M, Université MontPellier 2, F-34095, Montpellier Cedex 5, France

E-mail address: Claude.Cibils@math.univ-montp2.fr

Department of Mathematics, Shanghai Jiao Tong University, Shanghai 200240, PeoPLE'S REPUBLIC OF CHINA

E-mail address: pzhang@sjtu.edu.cn 